\title{
Multi-User System for the Virtual Interaction of a Pasteurization Process
}

\author{
Carlos R. Solis, Alex P. Porras, Víctor H. Andaluz, and Jorge S. Sánchez \\ Universidad de las Fuerzas Armadas ESPE \\ Sangolquí-Ecuador \\ \{crsolis, apporras, vhandaluz1, jssanchez\}@espe.edu.ec
}

\begin{abstract}
This article presents the development of a virtual Multi-user system of an industrial process, of a pasteurizing plant. The system will have a realistic and intuitive environment, in order to train and train operators in tasks of visualization, monitoring and control, through immersion and interaction with the devices and instrumentation present in the industrial process. The development of the virtual environment is carried out by means of CAD design software for the modeling of all the elements together with the Unity 3D graphic engine, in addition to the MATLAB tool that provides information on the simulation of the process, while virtual reality devices are used for immersion. The characteristic of Multi-user is given by the creation of a server which synchronizes and allows the exchange of information between several users at the same time. The experimental tests allow the operator to interact with the virtual environment and acquire monitoring and control skills with the different stages of pasteurization of the dairy industry without risks and labor problems.
\end{abstract}

\section{CCS Concepts}

- Interaction of a pasteurizer plant $\rightarrow$ Virtual Reality - Multiple user system methodology $\rightarrow$ Users with different privileges -Dynamic modeling of the process $\rightarrow$ Virtual elements with real responses $\cdot$ Control of the pasteurization process $\rightarrow$ Virtual process tuning method.

\section{Keywords}

Virtual Reality; Unity3D; Multi-users; Control; Pasteurization.

\section{INTRODUCTION}

Virtual Reality (VR) is defined as a set of fused technologies, developed to integrate the user with virtually designed environments [1]. Virtual reality can also be described as the science that integrates man with information. [2]. The main objective is to generate sensations and experiences that are obtained in the real world, through input and output devices that allow interaction with the environments. These environments can be models of the real world or imaginary [3].
VR has become a field in a large number of applications in different areas such as: $i$ ) Medicine, which makes it possible to simulate or emulate treatments for burn pain and wound care [4]; ii) Education, through this technology, children, young people and adults are transported to a virtual world, thus developing their imagination and creativity. They improve their skills in the face of a possible fact, and it is the best way to evaluate the ability to deal with a real problem [5]; iii) Robotics presents animations of coupled and uncoupled robotic mechanisms, creates 3D simulators applied to the robotics area that allow analyzing the performance of different autonomous control schemes in different environments [6]; iv) Safety in nuclear facilities, since it has developed similar possible environments between real facilities and the virtual scenario [7]; $v$ ) Leisure and Entertainment is another field that has grown greatly since it created video games and computer simulations, since the objective of the simulation is to represent real-world aspects in a realistic manner [8]; vi) Industrial Area, a virtual system can serve as a useful and effective module for industrial training [9], it seeks to implement collaborative environments that facilitate the participation of several users in the development of processes where they acquire skills of control and monitoring of the processes of one or more variables and become familiar with the activities that will be carried out in real life. These virtual processes integrate real parameters and units, which are very effective in contributing knowledge to users. With a complete virtual environment, reliable design criteria and key points in quality maintenance can be given, in this way the virtualization of the equipment becomes a very useful and effective tool for maintenance and training processes [10]. A specialized virtual environment working in real time, can provide critical and high priority information of a process [11].

Nowadays, VR plays a very important role in the industry, since it allows to train professionals about the processes that are executed in them. Thus, the success of the industries depends in great part on the capacity of the work personnel, both to perform and improve their daily activities, as well as to solve technical inconveniences that arise in a process, due to human errors or technical or mechanical failures. With this technology, the limitations that form part of technical manuals and verbal explanations are set aside and the virtualization of processes is opened with the freedom to cause some damage to the process and some physical damage to personnel.

The performance of the professionals in the industry is determinant for the increase of efficiency, production and profits; the industry needs professionals who have good knowledge and great experience. This can be solved through an excellent training, which can be through a virtual reality system with Multi-user feature, where you can share knowledge and demonstrate the results before different actions. This is how the interest in developing virtual Multi-user systems has increased, which allow more than one user to access the same environment at the same time. Multi-user 
systems are a very effective option for technical, immersive and social learning. Through this type of system, it is possible to obtain a critical analysis of the results, in addition it improves the experience in the handling of the environment when including 3D models to the detail [12]. This type of systems can be exposed through web portals, which facilitates their use, allowing to participate in virtual tasks through a web browser commonly installed on any computer [13].

In order to improve the method of user training, this paper presents the development of a virtual reality system Multi-user of a pasteurizer plant. The components and instrumentation of the pasteurizing plant are developed based on technical visits, civil plans of the industry and its P\&ID diagram, using CAD design software. The animation of objects, as well as sounds and lighting is implemented in the Unity3D graphic engine. The dynamics and control of the processes present in the plant are simulated with the help of MATLAB by means of the transfer function. Finally, a mobile app is implemented that allows me to visualize, monitor and control the values of the variables of the processes, both temperature and level.

\section{SYSTEM STRUCTURE}

This document presents the development of an interactive virtual Multi-user training system for an industrial process of a milk pasteurization plant. The proposed system manages textures and movements in a virtual environment, which will be designed to develop monitoring, control and training skills of this industrial process free of labor risks, for this, the work is divided into 3 main stages as shown in Figure 1.



Figure 1. System structure diagram.

Industrial Process: This stage shows the digitalization of the pasteurization process of the company El Ranchito S.A for which the modeling of the existing industrial instrumentation in the pasteurization processes is carried out, by means of CAD design software and the graphic engine Unity3D. All these processes will be carried out based on the industrial safety standards that the company manages. Two industrial processes intervene in the milk pasteurization plant: level and temperature, from these processes the dynamics of each one is obtained and the control algorithms are created in a closed loop in order to execute monitoring and control tasks.

The data exchange of physical variables is done with the help of MATLAB with the respective mathematical modeling, which causes a certain behavior of the variables in the virtual environment. The realism and immersion of the system is granted by the virtual reality devices, which facilitates to the users the interaction with the process, the models that are obtained are of elements with similar characteristics to those that exist in the processes of pasteurization of the company Ranchito SA.

Communication: This stage manages the exchange of information between the server, which is synchronized with all users and the database which has the information processed before, so it is given the feature of two-way communication. It should be borne in mind that any modification or activation of a user that occurs in the virtual environment of the plant, will be displayed and can be viewed by all users connected.

Users: At this stage is the creation of the Multi-user System which is done using the accents of Unity 3D which achieves coordinated hierarchical implementation between server and clients, and thus synchronizes different users in the same virtual environment. The communication between multiple users is at the same time, in addition each user is defined by means of ranks and privileges that will be granted to him. Ranks and privileges are created based on an access level SCADA system.

\section{3D VIRTUALIZATION}

This section describes the process used for the virtualization of the milk pasteurization plant, through the modeling of devices and instrumentation present in the plant, with CAD design software and the Unity 3D graphic engine, through access to P\&ID diagrams, technical visits and civil construction plans, as well as avatar design, animations and auditory effects that provide greater immersion in the training environment. The virtual Multi-user system for training and capacitation of a milk pasteurization plant, has been built through a sectional scheme in order to achieve the necessary immersion and interaction in a virtual environment that fits reality, see Figure 2. Thus, we have the following phases:

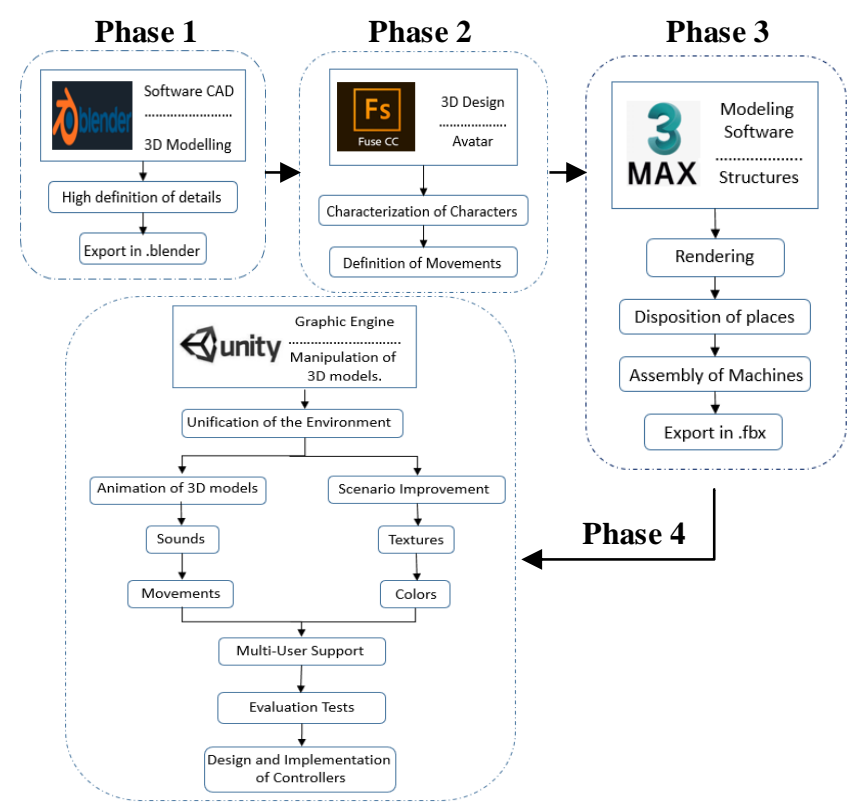

Figure 2. Multi sections of virtualization.

For the implementation of a VR system it is necessary to integrate a certain number of components and parameters, this process is described in Phase 1, which consists of 3D modeling of the elements that make up the virtual environment, where a CAD design software is used, because this tool allows creating $3 \mathrm{D}$ models identical to reality, considering the physical and mechanical characteristics of those that are part of the pasteurizer, the tool used is Blender because it has the necessary characteristics in a single package.

It is very important to implement the avatar in a VR system, which is described in Phase 2. The fact of having an avatar is to look and act like the user, but within a virtual space, so through the Adobe 
Fuse software, characteristic for its convenience of development, the avatars were designed, which differ by dress and the type of access they are granted, i.e., a SCADA system was applied for user access, where each has different privileges.

The plans of the civil infrastructure are necessary to make a virtual survey the most similar of the industry, where walls, doors, windows, etc. are detailed, that is why the importance of Phase 3. For this section is possible and can edit the elements is used the software 3DS Max, because it is a widely used tool due to the efficiency and flexibility it has. All the objects are ready to be imported into Unity 3D in a *.FBX file. Finally, we have the Phase 4 that is given once constructed, modified and conditioned the 3D models, these are exported to the graphical engine of Unity3D to make the programming that provide animation and realism to the objects of the program, and allow the user to interact with the virtual medium. These animations include the visualization of the level of the milk reception, mixing and storage tanks activation of alarms and color change in devices, indicating their on and off, see Figure 3.

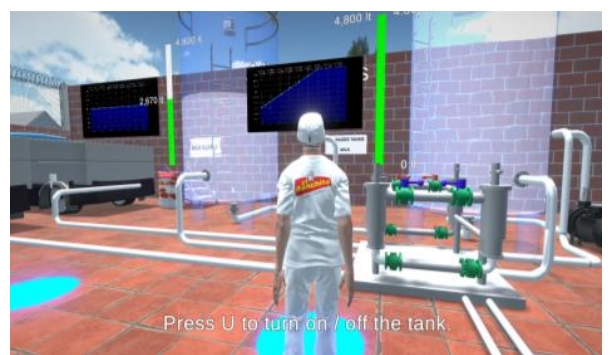

Figure 3. Integration to Unity 3D graphic platform.

\section{MODELLING AND CONTROL}

This section presents the development of the mathematical modeling of the level and temperature processes present in the milk pasteurizer plant and the design of the control algorithms that execute the simulation of the processes through the MATLAB software. To obtain the mathematical modeling of the processes present in the pasteurization, instruments similar to those of the company Ranchito S.A. were used, in addition to the software TIA PORTAL, LabVIEW and the PLC S7-1200 device.

\subsection{Mathematical modeling of the systems}

To obtain the mathematical modeling of the processes present in pasteurization, it was decided to use a level plant and a temperature plant with their respective P\&ID diagrams. This article presents the development of the level process (see Figure 4.), which uses a development methodology similar to the temperature plant shown in Figure 5.

The level process of the pasteurizer plant consists of three tanks and a pump, which in the P\&ID diagram is represented as follows: milk receiving tank and mixing tank as tank 1 ; storage tank as tank 2 and the pump which is represented in the same way.

To obtain the dynamics of the process, data was recorded at a step input, by means of the response between the set point (CO) and the filling value of the tank in the process (PV), achieving the following curves, see Figure 6.

Figure 6 also shows the relationship between the voltage applied to the frequency converter (CO) and the output flow rate (PV), which provides information on the actual behavior of the plant.

The level process is an integral system; therefore, the analysis and calculation of the mathematical model is made from equation 1.

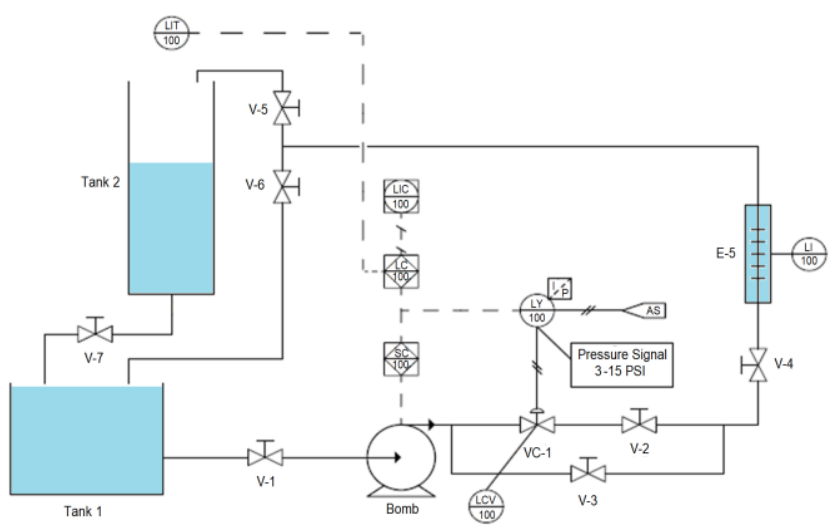

Figure 4. Plant level P\&ID diagram.

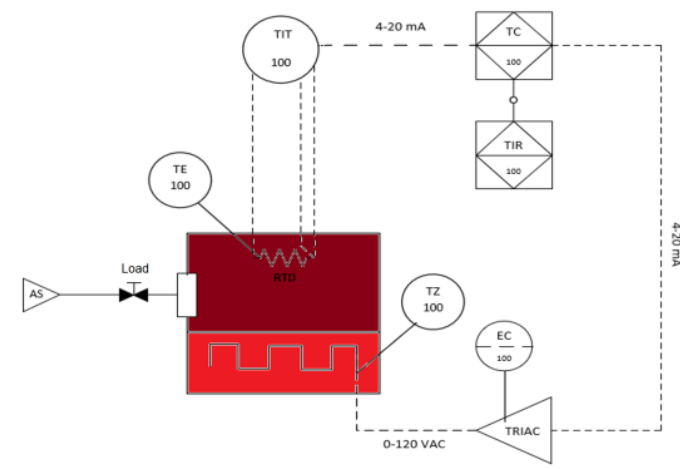

Figure 5. Plant temperature P\&ID diagram.



Figure 6. Inlet curves and milk tank filling response.

$$
G_{m}(s)=\frac{K_{m} e^{-T_{m} s}}{s}
$$

The data for this equation are obtained from the curves resulting from the previously recorded SP and PV data, i.e. from the behavior of the process.

$$
\begin{gathered}
K_{m}=\frac{O_{2}-O_{1}}{\left(I_{2}-I_{1}\right)\left(T_{3}-T_{2}\right)} \\
K_{m}=0.058 \\
T_{m}=\left(T_{2}-T_{1}\right) \\
T_{m}=2.6
\end{gathered}
$$

As a result, we have the mathematical model of the level process, which is described by: 


$$
G_{m}(s)=\frac{5,085 \times 10^{-3} e^{-2,6 s}}{s}
$$

For the proper and efficient behavior of the controller is necessary to tune and configure it with the best result, for this we have opted for the following methods and have obtained the following results. Different methods are used to tune the controller as detailed in Table 1.

Table 1. Tuning Methods

\begin{tabular}{|c|c|c|}
\hline Ford Method & Armstrong Method & Hay Method \\
\hline$K_{P}=\frac{1.48}{K_{m} \times T_{m}}=11.25$ & $K_{P}=\frac{0.94}{K_{m} \times T_{m}}=7.116$ & $K_{P}=\frac{0.4}{K_{m} \times T_{m}}=3.028$ \\
$T_{i}=2 T_{m}=5.2$ & $T_{i}=2 T_{m}=5.2$ & $T_{i}=3.2 T_{m}=8.32$ \\
$T_{d}=0.37 T_{m}=0.962$ & $T_{d}=0.5 T_{m}=1.3$ & $T_{d}=0.8 T_{m}=2.08$ \\
\hline
\end{tabular}

As a result of the Ford method we obtain the following control system, see Figure 7.



Figure 7. Ford Method Control System.

As a result of the Angstrom method we obtain the following control system, see Figure 8.

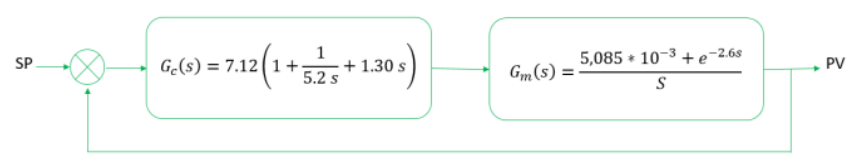

Figure 8. Angstrom method control system.

As a result of the Hay method we obtain the following control system, see Figure 9.

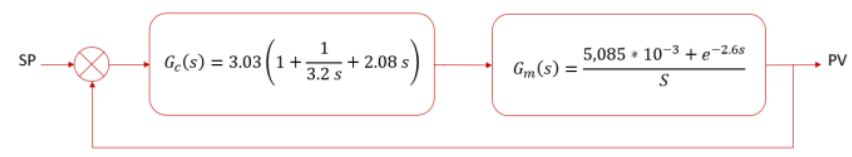

Figure 9. Hay method control system.

Figure 10. Shows the behaviour of each of the tuning methods, which when subjected to a certain step the response follows a certain curve until it reaches its final value, all this depends on the equations that have been raised.

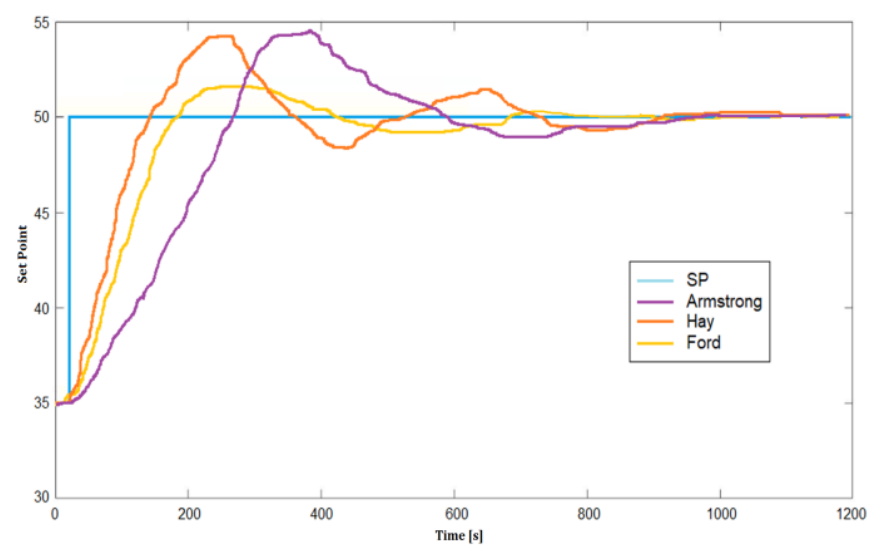

Figure 10. Dynamic behaviour in each response.

The temperature process is a self-regulating system; therefore, the analysis and calculation of the mathematical model is made from equation 7 .

$$
G p(S)=\frac{K p e^{-\theta p S}}{\tau p S+1}
$$

Data for these variables is calculated with the help of MATLAB software, and tuning is performed using the following methods: Ziegler-Nichols; Lambda; Halman; and Cohen-Coon.

For the simulation and control of the milk level process, in the storage tanks it is required the integration of the mathematical model natively in the server, this is possible through the creation and programming of a script (PID Controller) which will allow the exchange of data and information between all online users, and process control, (see Fig. 11), for which we have developed the design of the HMI's and the relevant programs in each of them.

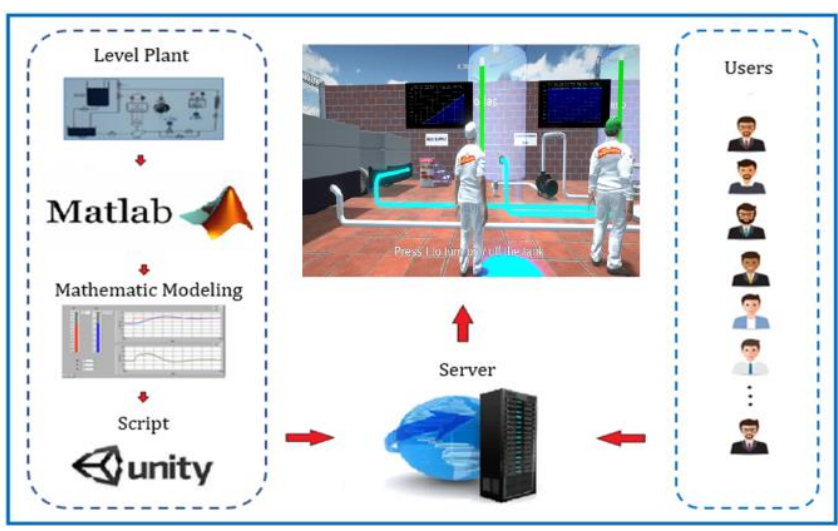

Figure 11. Diagram of data exchange between applications.

\section{MULTI-USER SYSTEM}

The implementation of the multi-user system is done through Unity3D together with the Internet, where it has information from a database that communicates bidirectionally in a synchronized way between the different users, which will allow us to access and interact with the process from anywhere. Next, the Figure 12, shows the detailed scheme on which this system is based.

Figure 12. indicates that the Multi-user system was implemented through a Photon Unity Networking framework for games and multiplayer applications in real time; also, through the scripts in Unity, the creation of a Server; which is an instance with authority to coordinate all events. He himself is in charge of encapsulating the data and through remote procedure calls (RPC) exchange data sent by TCP/IP protocol through Photon SDK to communicate with the Web Services and update the data of the levels of tanks, pump drives, users, which has the database. The system has a login using Web Services, which were implemented in Google Cloud Platform and using a database created in FireBase.

By means of scripts, the model of the real dynamic behavior of the tanks and other elements that intervene in the pasteurization process is added; in addition, the script (PID Controller) acts as a level and temperature controller, see Figure 13; the same one that updates control data, in the variables that the script has (Tanks Controller), to be later visualized in the virtual environment (Levels Visualization) and (Current Process Visualization).

In addition, an Android application of the pasteurization process is designed, which acts as a remote plant for mobile devices see Figure 15; which allows us to have a mobile visualization, and manipulate process control parameters. 




Figure 12. Multi-user system structure diagram.

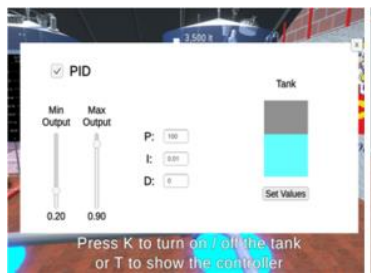

(a)PID Level

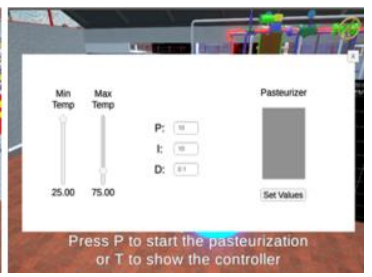

(b) PID Temperature
Figure 13. Control window.

The server is created in Unity3D by means of Photon server SDK, which allows to develop a Multi-user system, with Photon an authoritarian, persistent, preventive and predictive logic was implemented in a central instance among a number of clients [14].



Figure 14. Android application process window.

The server is the fundamental part of this work, here the whole system was implemented, from the most basic thing as the textures, happening through the PID controllers, model of the dynamics of the real behavior of the elements, up to the synchronization of data for the Multi-user system. The exchange of information between users is intercepted by the server, which decides what action to take, all this based on the programming structure it has. For this reason, the server acts as an intermediary between the users or clients and the database. The platform has an interesting client/server architecture that allows us to customize or add new custom servers. For ease of communication users can do it via audio, see Figure 15.

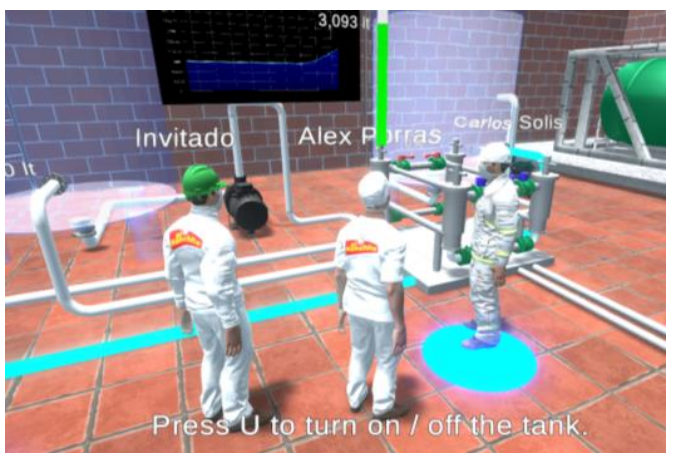

Figure 15. Multi-user interaction of milk storage tank filling.

Design of user levels and privileges: The Multi-user virtual system creates a 3D information environment that allows a series of different applications, depending on the type of user. These collaborative applications are aimed at specific tasks performed by user types that are distinguished by a user name and password, in which privileges are assigned in scripts with conditional sentences according to the implementation logic. To start the application, the menu is established, in which the operator establishes a User and a Password, which depends on the level of access that has been granted.

At the El Ranchito S.A. pasteurizer factory, we have identified three potential types of users.

- Operators (technicians, mechanics, maintenance, some with experience in different machines), are responsible for handling all types of level changes, drives and controllers, have type of white clothing with cap and protective mask, see Figure 16. 


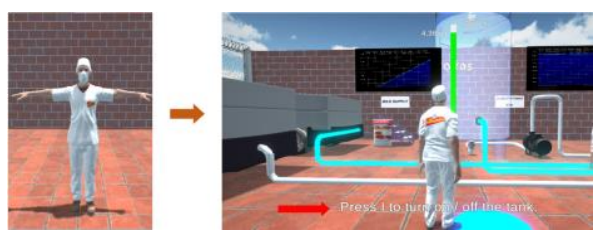

Figure 16. Avatar operator.

All this type of drive can be seen by the description that appears when approaching objects.

- $\quad$ Supervisors (they administer and decide on the needs of the company, in addition, they need data to maintain details of the plant), they are in charge of manipulating variables of the controllers of the process, in addition they administer the personnel has a registry of users for their entrance to the system (see Figure 17); they have type of white clothes rigid with white helmet and mask of protection.

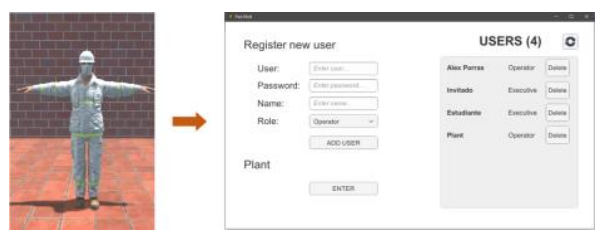

Figure 17. Avatar supervisor.

- Executives (including apprentices, suppliers, and customers). This user has the function of guest, which does not have privileges of changes and manipulation as they do not know the operation of the process, type of white clothing with green helmet and protective mask, see Figure 18.



Figure 18. Executive avatar.

This type of operator will not have access to any device throughout the tour of the pasteurizer plant, so you will not see any description.

\section{ANALYSIS OF RESULTS}

In order to evaluate the Multi-user application, it is proposed to run several experiments in which each object developed in the virtual environment is used.

To start the application, the menu is established, in which the operator establishes a User and a Password, which depends on the level of access that has been granted. Within the industrial pasteurization process there are 4 stages: reception of the milk; filtration and mixing of the milk; pasteurization; and storage of the final product, each of them contains a specific task. In these stages the user with operator access level can activate the valves, control the level of the tanks and vary the time of heating and cooling of the milk, with the presence of other users, one of them can be the supervisor of the part of production while the other users are executives or operators with the same tasks, each user has the possibility of intercommunicating between them to ask questions or ask for help with the task they are executing, see Figure 19.

Depending on the level of access granted to the users, they may or may not vary the set point of the process and the variables of the controller, in addition all users present may view the curves resulting from the controller.

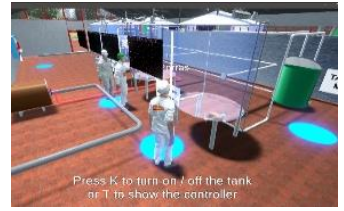

a) Pasteurization Area

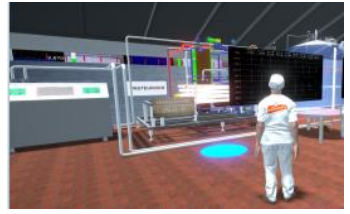

b) Storage Area Figure 19. Multi-user interaction.

All stages of pasteurization are designed to be Multi-user, here you can interact between the three types of users that were designed. For a better experience in training and training the system, communication between users is visual and auditory, i.e., all users can speak and listen at the same time. The total number of users is defined, opting for a maximum of eight people, which can be created or eliminated by the supervisor, due to the privilege granted in the access level.

This Multi-user system allows all users to share the same resources simultaneously, but generates a delay between drives of $4000 \mathrm{~ms}$ with a standard deviation of approximately +-5 . That is to say, there is a small delay time in sending and receiving the information between the users.

On the other hand, we have control of the processes that were given to the Multi-user virtual system, defined by the mathematical modeling that was obtained from the plant. As a result, we have an adequate and efficient behavior when tuning it through Ford's method, see Figure 20.

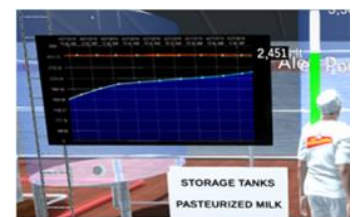

a) Set Point establishment



b) System clearing



c) Process control

Figure 20. Behaviour of the controller in the level process.

Figure 20 shows the behavior of the controller against an input variable (Set Point), in this case it is necessary to maintain the level of the tank at 2450 liters. In order to maintain the level at the required value, the pump is activated if necessary, which allows the access of milk from the reception tanks to the mixing tank.

Next, the efficiency in the usability of virtual environments to carry out an industrial process is indicated, in our case: the pasteurization of milk. To achieve this, we use the SUS summary evaluation method [15], in which we obtain a Likert style scale [16] that generates a unique value, represented by a mean composed by the usability of the global system under study.

There are 10 selected questions, see Table 2. The selected points have a correlation between 0.7 and 0.9 . The score ranges from 1 meaning a complete disagreement to 5 being completely in agreement. To calculate the SUS score, you must first add the contributions of each point that contribution will range from 0 to 4 . 
For the numbers 1,3,5,7 and 9, the contribution will be the position of the scale minus 1 . For the points $2,4,6,8$ and 10 , the contribution will be 5 minus the position in the scale. The sum of the results is multiplied by 2.5 to give the overall value of the SUS. The result will be in the range of 0 and 100 . For the answers indicated to the questions, the following table is obtained.

Table 2: Questionnaire results

\begin{tabular}{lcc}
\hline \multicolumn{1}{c}{ Questions } & Score & Operation \\
\hline $\begin{array}{l}\text { I consider this system to be very user- } \\
\text { friendly. }\end{array}$ & 4 & $4-1=3$ \\
$\begin{array}{l}\text { How complex was the handling of a } \\
\text { pasteurization process in a virtual } \\
\text { environment? }\end{array}$ & 2 & $5-2=3$ \\
$\begin{array}{l}\text { I think that prior to the use of the system an } \\
\text { induction would be needed? }\end{array}$ & 5 & $5-1=4$ \\
$\begin{array}{l}\text { I think I would need technical support to } \\
\text { make use of the system? }\end{array}$ & 1 & $5-1=4$ \\
$\begin{array}{l}\text { I consider that the experience in the virtual } \\
\text { environment is very similar to reality? }\end{array}$ & 5 & $5-1=4$ \\
$\begin{array}{l}\text { Have I found too much inconsistency in } \\
\text { this system? }\end{array}$ & 1 & $5-1=4$ \\
$\begin{array}{l}\text { I think most industrial processes can be } \\
\text { brought to virtual reality? }\end{array}$ & 4 & $4-1=3$ \\
$\begin{array}{l}\text { Do I find it uncomfortable to use the } \\
\text { system to carry out an industrial process? }\end{array}$ & 1 & $5-1=4$ \\
$\begin{array}{l}\text { Have I felt very safe making use of the } \\
\text { system? }\end{array}$ & 5 & $5-1=4$ \\
$\begin{array}{l}\text { I believe that the delays in the system, } \\
\text { cause a malfunction of the pasteurization } \\
\text { processes or erroneous data? }\end{array}$ & 1 & $5-1=4$ \\
\hline \multicolumn{1}{c}{ Total }
\end{tabular}

The total obtained is 37 which multiplication by 2.5 , resulting in 92.5 which means that the software has high performance and is suitable for training users in the pasteurization process, feature of usability.

\section{CONCLUSIONS}

The virtualization of the milk pasteurization plant, by means of 3D modeling of the elements and instrumentation present in the process, allows to obtain a great experience in the immersion of the users with the virtual environment. The advantage of this is the training that the users obtain about the pasteurization process without exposing themselves to risks that are presented in the real process equipment. At the same time, they become a tool to familiarize themselves with the stages handled by the pasteurizing industry. The use of the developed system allows the personnel to have an approach to the control systems and allows a continuous, realistic and Multi-user training, thus the virtual system provides a useful knowledge to operators, supervisors and other users in the control of processes, which reduces at the same time labor risks.

Acknowledgements. The authors would like to thanks to the Corporación Ecuatoriana para el Desarrollo de la Investigación y Academia-CEDIA for the financing given to research, development, and innovation, through the CEPRA projects, especially the project CEPRA-XIII-2019-08; Sistema colaborativo de Robots Aéreos para Manipular Cargas con Optimo Consumo de Recursos; also to Universidad de las Fuerzas Armadas ESPE, Escuela Superior Politécnica de Chimborazo, Universidad Nacional de Chimborazo, Universidad Técnologica Indoamerica, Universidad Internacional de Ecuador, Universidad Central de Venezuela, Venezuela and Grupo de Investigación ARSI, for the support to develop this work.

\section{REFERENCES}

[1] M. Back, D. Kimber, E. Rieffel, D. Anthony, B. Liew, S. Gattepally, J. Foote, J. Shingu y J. Vaughan, The virtual chocolate factory: Building a real world mixed-reality system for industrial collaboration and control, de IEEE International Conference on Multimedia and Expo, Ciudad de Suntec, Singapur, 2010.

[2] C. SangSu, J. Kiwook y D. N. Sang, Virtual reality applications in manufacturing industries: Past research, present findings, and future directions, Concurrent Engineering: SAGE, pp. 1-24, 2015.

[3] D. Grajewski, F. Gorski, P. Zawadzki y H. Adam, Application of Virtual Reality Techniques in Design of Ergonomic Manufacturing Workplaces, International Conference on Virtual and Augmented Reality in Education, PROCEDIA, pp. 289-301, 2013.

[4] J. Ortiz, J. Sánchez, P. Velasco, C. Sánchez, W. Quevedo, V. Zambrano, O. Arteaga y V. Andaluz, Teaching-learning oricess through VR applied to automotive engineering., INternational Conference on Education Technology and Computer, pp. 36-40, 2017.

[5] V. Andaluz, A. Pazmiño, J. Perez, C. Carvajal, F. Lozada, J. Lascano y J. Carvajal, Training of Tannery Processes Through Virtual Reality, Springer, pp. 75-93, 2017.

[6] Andaluz, J. Sanchez, C. Sanchez, W. Quevedo, J. Varela, J. Morales y G. Cuzco, Multi-User Industrial Training and Education Environment, Springer-Verlag Berlin Heidelberg, pp. 1-3, 2011.

[7] G. Dini y M. Mura, Application of augmented reality techniques in through-life engineering services, Procedia CIRP 38, pp. 14-23, 2015.

[8] V. Elia, M. Gnoni y A. Lanzilotto, Evaluating the application of augmented reality devices in manufacturing from a process point of view, Expert Syst Appl. 63, pp. 187-197, 2016.

[9] S. Kim, H. Suk, J. Kang, J. Jung, T. Laine y J. Westlin, Using unity $3 \mathrm{D}$ to facilitate mobile augmented reality game development., IEEE World Forum on Internet of Things (WF-IoT), Seoul, pp. 21-26, 2014.

[10] T. Bogoni, R. Scarparo y M. Pinho, A virtual reality simulator for training endodontics procedures using manual files, IEEE Symposium on 3D User Interfaces (3DUI), pp. 39-42, 2015.

[11] H. Kan, D. Vincent G y J. S. Chuan, An Internet virtual reality collaborative environment for effective product design, Elsevier, vol. 45, no 2, pp. 197-213, 2001.

[12] P. Orduña, A. Almeida, D. López-De-Ipiña y J. GarciaZubia, Learning Analytics on federated remote laboratories, IEEE Global Engineering Education Conference EDUCON, pp. 299-305, 2014.

[13] V. Andaluz, J. Sanchez, J. Chamba, P. Romero, F. Chicaiza, J. Varela, W. Quevedo, C. Gallardo y L. Cepeda, Unity3D Virtual Animation of Robots with Coupled and Uncoupled Mechanism, International Conference on Augmented Reality, Virtaul Reality and Computer Graphics, vol. 9768, $\mathrm{n}^{\circ}$ ISBN 978-3-319-40621-3, pp. 89-101, 2016.R.

[14] D. Jing, S. Yangming, M. Chao y Q. John, Communication by Interaction: A Multiplayer VR Environment for Building Walkthroughs, Construction Research Congress, pp. 5-8, 2016.

[15] J. Sauro y J. R. Lewis, When designing usability questionnaries, does it hurt to be positive, In Proceeding of the SIGCHI Conference on Human Factors in Computing Systems, pp. 2215-2224, $2011 \mathrm{~V}$.

[16] R. Linkert, A technique for the measurement of attitudes, de Archives of paychology, 1932. 